\title{
Research Article \\ Strictly Decreasing Solutions of a Class of Iterative Equations on the Unit Circle
}

\author{
Wei Song, Sheng Chen, and Xunbo Yin \\ Department of Mathematics, Harbin Institute of Technology, Harbin 150001, China \\ Correspondence should be addressed to Wei Song,dawenhxi@126.com
}

Received 22 February 2010; Revised 5 July 2010; Accepted 2 August 2010

Academic Editor: Manuel De la Sen

Copyright (C) 2010 Wei Song et al. This is an open access article distributed under the Creative Commons Attribution License, which permits unrestricted use, distribution, and reproduction in any medium, provided the original work is properly cited.

We discuss the strictly decreasing solutions of a class of iterative equations on the unit circle $\mathbb{T}^{1}$. The conditions for the existence, uniqueness, and stability of such solutions are presented.

\section{Introduction}

Let $C^{0}(X, X)$ be the set of all continuous self-mappings on a topological space $X$. Let $f \in$ $C^{0}(X, X)$. For integer $n \geq 0$, define the $n$th iterate of $f$ by $f^{n}=f \circ f^{n-1}$ and $f^{0}=\mathrm{id}$, where id denotes the identity mapping on $X$ and o denotes the composition of mappings. An equation with iteration as its main operation is simply called an iterative equation. Founded on the problem of iterative roots, the problem of invariant curves and some problems originating from dynamical systems, and the iterative equation

$$
\Phi\left(f(x), f^{2}(x), \ldots, f^{n}(x)\right)=F(x), \quad x \in X
$$

where $F$ and $\Phi$ are given functions and $f$ is the unknown function, have been investigated extensively $[1,2]$.

Let $\mathbb{T}^{1}=\left\{z \in \mathbb{C}: z=e^{2 \pi i t}, t \in \mathbb{R}\right\}$ denote the unit circle and $C^{0}\left(\mathbb{T}^{1}, \mathbb{T}^{1}\right)$ denote the set of all continuous maps from $\mathbb{T}^{1}$ to itself. Let 1 denote the point $(1,0)$ in the complex plane $\mathbb{C}$ so as to distinguish it from $1 \in \mathbb{R}$. The iteration on the unit circle $\mathbb{T}^{1}$ is also important. Many scholars had got a lot of results on iterative roots and iteration groups, such as [3-7]. In 2007, Zdun and Zhang [8] discussed the solutions of (1.1) on $\mathbb{T}^{1}$, that is, the equation

$$
\Phi\left(f(z), f^{2}(z), \ldots, f^{n}(z)\right)=F(z), \quad z \in \mathbb{T}^{1}
$$


in the class of homeomorphisms

$$
H_{1}^{0}\left(\mathbb{T}^{1}, \mathbb{T}^{1}\right)=\left\{f \in C^{0}\left(\mathbb{T}^{1}, \mathbb{T}^{1}\right): f\left(\mathbb{T}^{1}\right)=\mathbb{T}^{1} \text { homeomorphically and } f(\mathbf{1})=\mathbf{1}\right\}
$$

In [8], the authors lift $F$ from the circle $\mathbb{T}^{1}$ to $\mathbb{R}$ and $\Phi$ from the $n$-dimensional torus $\mathbb{T}^{n}$ to $\mathbb{R}^{n}$. They apply ingenious techniques of restricting and extending to these lifts so that the problem can be reduced and discussed on the compact interval $[0,1]$. However, they only discussed the strictly increasing solutions of (1.2). In this paper, we will discuss the strictly decreasing solutions of a special form of (1.2), that is, the following equation:

$$
\Phi\left(f(z), f^{3}(z), \ldots, f^{2 n-1}(z)\right)=F(z), \quad z \in \mathbb{T}^{1}
$$

\section{Basic Definitions and Lemmas}

As in [8], let $h: t \in \mathbb{R} \mapsto e^{2 \pi i t} \in \mathbb{T}^{1}$. If $v, w, z \in \mathbb{T}^{1}$, there exist unique $t_{1}, t_{2} \in[0,1)$ such that $w h\left(t_{1}\right)=z$ and $w h\left(t_{2}\right)=v$. The cyclic order on $\mathbb{T}^{1}$ is defined in [9], that is,

$$
\begin{gathered}
v \prec w \prec z \Longleftrightarrow 0<t_{1}<t_{2} \\
v \leq w \leq z \Longleftrightarrow t_{1} \leq t_{2} \text { or } t_{2}=0 .
\end{gathered}
$$

Obviously, the relations $v \prec w \prec z, w \prec z \prec v$ and $z \prec v \prec w$ are equivalent.

Considering a nonempty subset $A \subseteq \mathbb{T}^{1}$, a map $f: A \rightarrow \mathbb{T}^{1}$ is called to be increasing (strictly increasing) if $f(v) \leq f(w) \leq f(z)(f(v) \prec f(w) \prec f(z)$, resp.) for all $v, w, z \in A$ with $v<w<z$. Similarly, a map $f: A \rightarrow \mathbb{T}^{1}$ is called to be decreasing (strictly decreasing) if $f(z) \preceq f(w) \preceq f(v)(f(z) \prec f(w) \prec f(v)$, resp.) for all $v, w, z \in A$ with $v \prec w \prec z$. A homeomorphism $h: \mathbb{T}^{1} \rightarrow \mathbb{T}^{1}$ is said to be orientation preserving (orientation reversing) if it is strictly increasing (strictly decreasing, resp.).

If $v, z \in \mathbb{T}^{1}$ with $v \neq z$, there exist $t_{v}, t_{z} \in \mathbb{R}$ with $t_{v}<t_{z}<t_{v}+1$ and $h\left(\mathrm{t}_{v}\right)=v, h\left(t_{z}\right)=z$. Define the oriented arc

$$
\overrightarrow{(v, z)}=\left\{w=h(t): t \in\left(t_{v}, t_{z}\right)\right\}
$$

Obviously, the map $f$ is strictly increasing (strictly decreasing) if $w \in \overrightarrow{(v, z)}$ yields $f(w) \in$ $\overrightarrow{(f(v), f(z))}(f(w) \in \overrightarrow{(f(z), f(v))}$, resp.).

\subsection{The Lifts of Maps on $\mathbb{T}^{1}$}

Let $f \in C^{0}\left(\mathbb{T}^{1}, \mathbb{T}^{1}\right)$. A continuous map $\tilde{f}: \mathbb{R} \rightarrow \mathbb{R}$ is said to be a lift of $f$ if

$$
h \circ \tilde{f}=f \circ h .
$$

The following properties are well known. 
Lemma 2.1 (see [10]). (i) Each $f \in C^{0}\left(\mathbb{T}^{1}, \mathbb{T}^{1}\right)$ has a lift $\tilde{f}$. (ii) There exists a constant $k \in \mathbb{Z}$ such that each lift $\tilde{f}$ of $f$ satisfies $\tilde{f}(t+1)-\tilde{f}(t)=k$, for all $t \in \mathbb{R}$. The constant $k$ is said to be the degree of $f$ and denoted by $\operatorname{deg}(f)$, that is, $\operatorname{deg}(f)=\tilde{f}(t+1)-\tilde{f}(t)$, for all $t \in \mathbb{R}$. (iii) For $f, g \in C^{0}\left(\mathbb{T}^{1}, \mathbb{T}^{1}\right)$ and id $: \mathbb{T}^{1} \rightarrow \mathbb{T}^{1}$, one has $\operatorname{deg}(g \circ f)=\operatorname{deg}(g) \cdot \operatorname{deg}(f)$ and $\operatorname{deg}($ id $)=1$. If $f \in C^{0}\left(\mathbb{T}^{1}, \mathbb{T}^{1}\right)$ is a homeomorphism, then $|\operatorname{deg}(f)|=1$. (iv) If $\tilde{f}$ is a lift of $f$, then for each $j \in \mathbb{Z}$, the map $\tilde{f}+j$ is a lift of $f$ and every lift of $f$ can be expressed in this form, where $\mathbb{Z}$ denotes the set of integers.

Lemma 2.2 (see [10]). (i) If $g:[0,1] \rightarrow \mathbb{R}$ is a continuous map such that $g(1)-g(0)$ is an integer $k$, then $g$ can be extended to a map $\tilde{g}: \mathbb{R} \rightarrow \mathbb{R}$ with

$$
\tilde{g}(t+1)-\tilde{g}(t)=k, \quad \forall t \in \mathbb{R}
$$

(ii) Suppose $\tilde{f}: \mathbb{R} \rightarrow \mathbb{R}$ is continuous, $k$ is an integer, and $\tilde{f}(t+1)-\tilde{f}(t)=k$, for all $t \in \mathbb{R}$. Then there exists a unique $f \in C^{0}\left(\mathbb{T}^{1}, \mathbb{T}^{1}\right)$ such that $\tilde{f}$ is a lift of $f$.

A map $f \in C^{0}\left(\mathbb{T}^{1}, \mathbb{T}^{1}\right)$ is said to be Lipschitzian if there exists a lift $\tilde{f}$ of $f$ satisfying

$$
\left|\tilde{f}\left(t_{1}\right)-\tilde{f}\left(t_{2}\right)\right| \leq M\left|t_{1}-t_{2}\right|, \quad \forall t_{1}, t_{2} \in \mathbb{R}
$$

for a constant $M>0$. By (iv) of Lemma 2.1, the constant $M$ is independent of the choice of $\tilde{f}$, and $M$ is said to be a Lipschitzian constant of $f$.

In the sequel one will discuss the strictly increasing (strictly decreasing) maps $f \in$ $C^{0}\left(\mathbb{T}^{1}, \mathbb{T}^{1}\right)$ by means of their lifts.

Lemma 2.3. A lift $\tilde{f}$ of $f \in C^{0}\left(\mathbb{T}^{1}, \mathbb{T}^{1}\right)$ is strictly monotonic (monotonic) if and only if there exists a $t_{0} \in \mathbb{R}$ such that $\left.\widetilde{f}\right|_{\left[t_{0}, t_{0}+1\right)}$ is strictly monotonic (monotonic).

The proof is trivial. So we omit it.

Lemma 2.4 (see [11]). A map $f \in C^{0}\left(\mathbb{T}^{1}, \mathbb{T}^{1}\right)$ is strictly increasing (strictly decreasing) if and only if $\operatorname{deg}(f)=1(\operatorname{deg}(f)=-1)$ and its lift $\tilde{f}$ is strictly increasing (strictly decreasing) in $\mathbb{R}$.

Thus we get the following theorem.

Theorem 2.5. A map $f \in C^{0}\left(\mathbb{T}^{1}, \mathbb{T}^{1}\right)$ is strictly increasing (strictly decreasing) if and only if it is an orientation-preserving (orientation-reversing) homeomorphism.

Proof. We only need to show that $f$ has an inverse mapping. Let $\tilde{f}$ be the lift of $f$. The map $f^{-1}=h \circ \tilde{f}^{-1}$ is the inverse mapping, where $\tilde{f}^{-1}$ is the inverse function of $\tilde{f}$.

\subsection{Induced Maps of Maps on $\mathbb{T}^{1}$}

Firstly, we define

$$
H_{1}^{0}\left(\mathbb{T}^{1}, k\right)=\left\{f \mid f \in H_{1}^{0}\left(\mathbb{T}^{1}, \mathbb{T}^{1}\right), \operatorname{deg}(f)=k\right\},
$$


where $k=1$ or -1 . Let $h_{*}:=\left.h\right|_{(0,1)}$; the map $h_{*}$ is a homeomorphism from $(0,1)$ to $\mathbb{T}^{1} \backslash\{\mathbf{1}\}$. For $f \in H_{1}^{0}\left(\mathbb{T}^{1},-1\right)$, define $\tilde{f}_{*}:=h_{*}^{-1} \circ f \circ h_{*}$ which is a self-map on $(0,1)$. We extend $\tilde{f}_{*}$ to the compact interval $I=[0,1]$. Let

$$
G(t)= \begin{cases}1, & t=0, \\ \tilde{f}_{*}(t), & t \in(0,1), \\ 0, & t=1 .\end{cases}
$$

For convenience, we call $G$ the induced map of $f$, which is a self-map on $[0,1]$.

Lemma 2.6. The induced map $G$ of an orientation-reversing homeomorphism $f \in H_{1}^{0}\left(\mathbb{T}^{1},-1\right)$ is continuous and strictly decreasing on I. It can be extended to a lift $\tilde{f}$ of $f$. This lift satisfies $\tilde{f}(0)=$ $1, \tilde{f}(1)=0$ and is unique on this property.

Proof. Obviously, $G$ is continuous on the interval $(0,1)$. We only need to prove $\lim _{t \rightarrow 0^{+}} G(t)=1$ and $\lim _{t \rightarrow 1^{-}} G(t)=0$.

Concerning the continuity at 0 , we note that

$$
\lim _{t \rightarrow 0^{+}} G(t)=\lim _{t \rightarrow 0^{+}} h_{*}^{-1} \circ f \circ h_{*}(t)=\lim _{t \rightarrow 0^{+}} h_{*}^{-1} \circ f\left(e^{2 \pi i t}\right) .
$$

By the continuity of $f$ at $\mathbf{1}$, we have $\lim _{t \rightarrow 0^{+}} f\left(e^{2 \pi i t}\right)=\mathbf{1}$. Let $\widetilde{F}$ be any lift of $f$. By $h \circ \widetilde{F}=f \circ h$, we must have $\widetilde{F}(0)=k_{0} \in \mathbb{Z}$, where $\mathbb{Z}$ denotes the set of integers. By (iv) of Lemma 2.1, let $\widetilde{F}$ be a lift of $f$ with $\widetilde{F}(0)=1$. Since $f \circ h_{*}(t)=h_{*} \circ \tilde{F}(t), t \in(0,1)$, we have

$$
\lim _{t \rightarrow 0^{+}} h_{*}^{-1} \circ f \circ h_{*}(t)=\lim _{t \rightarrow 0^{+}} \tilde{F}(t)=1
$$

We can easily verify $\lim _{t \rightarrow 1^{-}} G(t)=0$ in the same way. Note that $G$ is strictly decreasing on $(0,1)$, so $G$ is strictly decreasing on $[0,1]$.

Let $t \in \mathbb{R}$, and $k$ is the integer with $t \in[k, k+1)$. Define $\tilde{f}:=G(t-k)+k$. We can easily get that $f \circ h=h \circ \tilde{f}$. Suppose that $\tilde{g}$ is another lift of $f$ which maps $[0,1]$ into itself and $\tilde{g}(0)=1, \tilde{g}(1)=0$. By (iv) of Lemma 2.1, we have $\tilde{f}=\tilde{g}+j$ for some integer $j$. Obviously, $j=\tilde{f}(0)-\tilde{g}(0)=0$. Then $\tilde{f}=\tilde{g}$.

The following lemma is a converse of Lemma 2.6.

Lemma 2.7. Suppose that $G \in C^{0}(I, I)$ is strictly decreasing and satisfies $G(0)=1, G(1)=0$. Then the map

$$
f:= \begin{cases}h_{*} \circ G \circ h_{*}^{-1}(z), & z \in \mathbb{T}^{1} \backslash\{\mathbf{1}\} \\ \mathbf{1}, & z=\mathbf{1}\end{cases}
$$

belongs to the class $H_{1}^{0}\left(\mathbb{T}^{1},-1\right)$. Moreover, $G$ can be extended to a lift of $f$. 
Proof. Clearly, $f(\mathbf{1})=\mathbf{1}$, and $\left.f\right|_{\mathbb{T}^{1} \backslash\{\mathbf{1}\}}$ is an orientation-reversing homeomorphism of $\mathbb{T}^{1} \backslash\{\mathbf{1}\}$. Then $f$ is orientation reversing on the whole $\mathbb{T}^{1}$. We only need to show the continuity of $f$ at 1. Let $z \rightarrow \mathbf{1}^{+}\left(z \rightarrow \mathbf{1}^{-}\right)$denote $z$ approaches to $\mathbf{1}$ in clockwise (counter-clockwise) direction. Note that

$$
\lim _{z \rightarrow 1^{+}} f(z)=\lim _{t \rightarrow 0^{+}} h_{*} \circ G \circ h_{*}^{-1}\left(e^{2 \pi i t}\right)=\lim _{t \rightarrow 0^{+}} h_{*} \circ G(t)=\mathbf{1} .
$$

By the same argument, we have $\lim _{z \rightarrow 1^{-}} f(z)=1$.

Given $t \in \mathbb{R}$ and integer $k$ with $t \in[k, k+1)$, define

$$
\tilde{f}(t)=G(t-k)+k
$$

One can easily show that $f \circ h=h \circ \tilde{f}$.

\subsection{Induced Maps of Maps on $\mathbb{T}^{n}$}

For simplicity, let

$$
\begin{aligned}
& \mathbb{T}^{n}=\overbrace{\mathbb{T}^{1} \times \cdots \times \mathbb{T}^{1}}^{n}, \\
& \mathbf{1}^{n}=(\overbrace{1, \ldots, 1}^{n}) .
\end{aligned}
$$

For $f \in H_{1}^{0}\left(\mathbb{T}^{1},-1\right)$, define

$$
H_{f}(z):=\left(f(z), f^{3}(z), \ldots, f^{2 n-1}(z)\right)
$$

Then (1.4) can be written as

$$
\Phi \circ H_{f}(z)=F(z), \quad z \in \mathbb{T}^{1}
$$

As in [8], we also have the following two remarks.

Remark 2.8. $H_{f}$ maps $\mathbb{T}^{1}$ into $\left(\mathbb{T}^{1} \backslash \mathbf{1}\right)^{n} \cup\left\{\mathbf{1}^{n}\right\}$.

Remark 2.9. Let $\operatorname{Rang}(\Phi)$ denote the range of $\Phi$. If $F \in H_{1}^{0}\left(\mathbb{T}^{1},-1\right)$ and (1.4) has a solution $f \in H_{1}^{0}\left(\mathbb{T}^{1},-1\right)$ on $F$, then Rang $\Phi=\mathbb{T}^{1}$ and $\Phi\left(\mathbf{1}^{n}\right)=\mathbf{1}$.

Let $\operatorname{Dom}(\Phi)$ denote the domain of $\Phi$. As in [8], $\operatorname{Dom}(\Phi)$ is very important for (1.4). Now, we discuss the relationship of $\operatorname{Dom}(\Phi)$ and (1.4). The first case is that the domain of $\Phi$ is $\mathbb{T}^{n}$. 


\subsubsection{The Case: $\operatorname{Dom}(\Phi)=\mathbb{T}^{n}$}

The following important and interesting lemma is proved in [8].

Lemma 2.10 (see [8]). If $\Phi: \mathbb{T}^{n} \rightarrow \mathbb{T}^{1}$ is continuous and $\Phi\left(\mathbf{1}^{n}\right)=\mathbf{1}$, then there exists a unique continuous function $\widetilde{\Phi}: \mathbb{R}^{n} \rightarrow \mathbb{R}$ such that

$$
\Phi\left(h\left(t_{1}\right), \ldots, h\left(t_{n}\right)\right)=e^{2 \pi i \tilde{\Phi}\left(t_{1}, \ldots, t_{n}\right)}, \quad \widetilde{\Phi}(0, \ldots, 0)=0 .
$$

Moreover, for each $k \in\{1, \ldots, n\}$, there exists an $m_{k} \in \mathbb{Z}$ such that

$$
\widetilde{\Phi}\left(t_{1}, \ldots, t_{k}+1, \ldots, t_{n}\right)=\widetilde{\Phi}\left(t_{1}, \ldots, t_{k}, \ldots, t_{n}\right)+m_{k}, \quad \forall t_{1}, \ldots, t_{k} \in \mathbb{R} .
$$

The map $\widetilde{\Phi}$ is said to be the lift of $\Phi$, and $\left(m_{1}, \ldots, m_{n}\right)$ is defined to be the degree of $\Phi$ and is denoted by $\operatorname{deg}(\Phi)$.

Lemma 2.11. Let $\widetilde{F}$ be the lift of $F$ with $\widetilde{F}(1)=0$, and let $\widetilde{\Phi}$ be the lift of $\Phi$ with $\widetilde{\Phi}(0, \ldots, 0)=0$. Let $f \in H_{1}^{0}\left(\mathbb{T}^{1},-1\right)$ be a solution of $(1.4)$, and let $\tilde{f}$ be a lift of $f$ with $\tilde{f}(1)=0$. Then (1.4) is equivalent to

$$
\tilde{\Phi}\left(\tilde{f}(t), \tilde{f}^{3}(t), \ldots, \tilde{f}^{2 n-1}(t)\right)=\tilde{F}(t), \quad t \in I
$$

Proof. Note that $f^{j}(h(t))=h\left(\tilde{f}^{j}(t)\right)$ for all $t \in \mathbb{R}$. For $z=e^{2 \pi i t}, t \in I$ and (1.4) is equivalent to

$$
\Phi\left(e^{2 \pi i \tilde{f}(t)}, e^{2 \pi i \tilde{f}^{3}(t)}, \ldots, e^{2 \pi i \tilde{f}^{2 n-1}(t)}\right)=e^{2 \pi i \widetilde{F}(t)} .
$$

By Lemma 2.10, $e^{2 \pi i \widetilde{\Phi}\left(\tilde{f}(t), \tilde{f}^{3}(t), \ldots, \tilde{f}^{2 n-1}(t)\right)}=e^{2 \pi i \tilde{F}(t)}$. This means that for each $t \in I$, we have

$$
\widetilde{\Phi}\left(\tilde{f}(t), \tilde{f}^{3}(t), \ldots, \tilde{f}^{2 n-1}(t)\right)=\tilde{F}(t)+k(t),
$$

where $k(t) \in \mathbb{Z}$. Since $\tilde{f}(1)=0, \widetilde{F}(1)=0$, and $\widetilde{\Phi}(0, \ldots, 0)=0$, we get $k(t)=0$. By the continuity of $\tilde{f}, \widetilde{F}$, and $\widetilde{\Phi}$, we get that $k(t) \equiv 0$.

Theorem 2.12. Suppose that $\Phi$ is continuous, $\Phi\left(\mathbf{1}^{n}\right)=\mathbf{1}, F$ is continuous, $F(\mathbf{1})=\mathbf{1}$, and (1.4) has a solution $f \in H_{1}^{0}\left(\mathbb{T}^{1},-1\right)$. Let $\operatorname{deg}(\Phi)=\left(m_{1}, \ldots, m_{n}\right)$. Then $\operatorname{deg}(F)=-\left(m_{1}+\cdots+m_{n}\right)$.

Proof. Let $\widetilde{F}$ be the lift of $F$ with $\widetilde{F}(1)=0$, and let $\widetilde{\Phi}$ be the lift of $\Phi$ with $\widetilde{\Phi}(0, \ldots, 0)=0$. Let $f \in H_{1}^{0}\left(\mathbb{T}^{1},-1\right)$ be the solution of $(1.4)$, and let $\tilde{f}$ be its lift with $\tilde{f}(1)=0$. Note that

$$
\tilde{f}(t+1)=\tilde{f}(t)-1, \quad t \in \mathbb{R} .
$$

Let $\widetilde{H}(t)=\left(\tilde{f}(t), \widetilde{f}^{3}(t), \ldots, \tilde{f}^{2 n-1}(t)\right)$. Since $\operatorname{deg}(\Phi)=\left(m_{1}, \ldots, m_{n}\right)$, then by (2.18), we have

$$
\widetilde{\Phi}(-1, \ldots,-1)=\widetilde{\Phi}(0, \ldots, 0)-m_{1}-\cdots-m_{n} .
$$


Thus, we get that

$$
\begin{aligned}
\widetilde{F}(t+1) & =\widetilde{\phi}(\widetilde{H}(t+1))=\widetilde{\Phi}(\widetilde{H}(t)+(-1, \ldots,-1)) \\
& =\widetilde{\Phi}(\widetilde{H}(t))-m_{1}-\cdots-m_{n} \\
& =\widetilde{F}(t)-m_{1}-\cdots-m_{n} .
\end{aligned}
$$

Definition 2.13. Suppose $\Phi: \mathbb{T}^{n} \rightarrow \mathbb{T}^{1}$ is continuous. Let $1 \leq i \leq n$, and let $w_{1}, \ldots, w_{i-1}$, $w_{i+1}, \ldots, w_{n} \in \mathbb{C}$ be fixed.

(1) One says $\Phi$ is increasing with respect to the $i$ th variable if the map

$$
\Phi\left(w_{1}, \ldots, w_{i-1}, z_{i}, w_{i+1}, \ldots, w_{n}\right)
$$

is increasing.

(2) One says $\Phi$ is constant with respect to the $i$ th variable if there is a $z_{0} \in \mathbb{T}^{1}$ such that the map

$$
\Phi\left(w_{1}, \ldots, w_{i-1}, z_{i}, w_{i+1}, \ldots, w_{n}\right) \equiv z_{0}
$$

Corollary 2.14. Let $F \in H_{1}^{0}\left(\mathbb{T}^{1},-1\right)$, and $\Phi$ is continuous with $\Phi\left(\mathbf{1}^{n}\right)=1$. If $\Phi$ is increasing with respect to each variable and $\Phi$ is nonconstant in at least two variables, then (1.4) has no solutions in $H_{1}^{0}\left(\mathbb{T}^{1},-1\right)$.

Proof. Let $\operatorname{deg}(\Phi)=\left(m_{1}, \ldots, m_{n}\right)$. Hence, by Lemma 2.3, we have $m_{1} \geq 0, \ldots, m_{n} \geq 0$ and $m_{k}=0$ if and only if $\Phi$ is constant with respect to the $k$ th variable. If (1.4) has a solution $f$ in $H_{1}^{0}\left(\mathbb{T}^{1},-1\right)$, then by Theorem 2.12 , we get that $\operatorname{deg}(F)=-\left(m_{1}+\cdots+m_{n}\right) \leq-2$. This a contradiction.

From the discussion above, we know that $\operatorname{deg}(F)$ and $\operatorname{deg}(\Phi)$ are interrelated. This is too severe for (1.4). So as in [8], by Remark 2.8, it is also natural to suppose that $\operatorname{Dom}(\Phi)=$ $\left(\mathbb{T}^{1} \backslash \mathbf{1}\right)^{n} \cup\left\{\mathbf{1}^{n}\right\}$.

\subsubsection{The Case $\operatorname{Dom}(\Phi)=\left(\mathbb{T}^{1} \backslash 1\right)^{n} \cup\left\{1^{n}\right\}$}

The following assumptions (H1) and (A) are cited from [8].

$(\mathrm{H} 1) \Phi:\left(\mathbb{T}^{1} \backslash\{\mathbf{1}\}\right)^{n} \cup\left\{\mathbf{1}^{n}\right\} \rightarrow \mathbb{T}^{1}$ is continuous, $\Phi\left(\left(\mathbb{T}^{1} \backslash\{\mathbf{1}\}\right)^{n}\right)=\mathbb{T}^{1} \backslash\{\mathbf{1}\}$.

(A) There exists a constant $\delta>0$ such that for $0<t_{k}<\delta, k=1, \ldots, n$, we have $\Phi\left(h_{*}\left(t_{1}\right), \ldots, h_{*}\left(t_{n}\right)\right) \in \overrightarrow{(\mathbf{1}, i)}$, and for $1-\delta<t_{k}<1, k=1, \ldots, n$, we have $\Phi\left(h_{*}\left(t_{1}\right), \ldots, h_{*}\left(t_{n}\right)\right) \in \overrightarrow{(-i, \mathbf{1})}$. 
Under assumptions (H1) and (A), as in [8], define

$$
\begin{gathered}
\Psi\left(t_{1}, \ldots, t_{n}\right):=h_{*}^{-1}\left(\Phi\left(h_{*}\left(t_{1}\right), \ldots, h_{*}\left(t_{n}\right)\right)\right), \quad t_{j} \in(0,1), j=1, \ldots, n, \\
\Psi(0, \ldots, 0)=0, \quad \Psi(1, \ldots, 1)=1 .
\end{gathered}
$$

The function $\Psi$ defined on $(0,1)^{n} \cup\{(0, \ldots, 0),(1, \ldots, 1)\}$ is said to be the induced map of $\Phi$. Let us note that

$$
\lim _{t_{j} \rightarrow 0, j=1, \ldots, n} \Psi\left(t_{1}, \ldots, t_{n}\right)=0, \quad \lim _{t_{j} \rightarrow 1, j=1, \ldots, n} \Psi\left(t_{1}, \ldots, t_{n}\right)=1
$$

Lemma 2.15 (see [8]). Under assumptions (H1) and (A), the induced map $\Psi$ of $\Phi$ is continuous.

Lemma 2.16. Let $\widetilde{F}$ be the lift of $F$ with $\widetilde{F}(1)=0$, and let $\Psi$ be the induced map of $\Phi$. Let $f \in$ $H_{1}^{0}\left(\mathbb{T}^{1},-1\right)$ be a solution of $(1.4)$, and let $\tilde{f}$ be a lift of $f$ with $\tilde{f}(1)=0$. Then (1.4) is equivalent to

$$
\Psi\left(\tilde{f}(t), \tilde{f}^{3}(t), \ldots, \tilde{f}^{2 n-1}(t)\right)=\tilde{F}(t), \quad t \in I
$$

Lemma 2.16 can be proved by means of the method which is used to prove Lemma 2.11.

As in [8], we also need the following assumption.

(H2) [8] There are nonnegative real constants $\alpha_{j}, \beta_{j}, j=1, \ldots, n$ with $\beta_{1} \geq \alpha_{1}>0, \beta_{j} \geq$ $\alpha_{j} \geq 0, j=2, \ldots, n$, such that

$$
\sum_{j=1}^{n} \alpha_{j}\left(t_{j}-s_{j}\right) \leq \Psi\left(t_{1}, \ldots, t_{n}\right)-\Psi\left(s_{1}, \ldots, s_{n}\right) \leq \sum_{j=1}^{n} \beta_{j}\left(t_{j}-s_{j}\right)
$$

for all $t_{j} \geq s_{j}$ in $(0,1), j=1, \ldots, n$.

Lemma 2.17 (see [8]). If $\Psi$ is differentiable in $(0,1)^{n}$ with respect to each variable and for every $k$ there exist $\alpha_{k}, \beta_{k}$ such that $\alpha_{1}>0,0 \leq \alpha_{k} \leq \partial \Psi / \partial t_{k} \leq \beta_{k}$, then $\Psi$ satisfies (H2).

\section{Existence of Strictly Decreasing Solutions}

Let $C^{0}(I)$ denote the set of all continuous maps on $I=[0,1]$. It is well known that $C^{0}(I)$ equipped with the supremum norm $\|g\|=\sup \{|g(t)|: t \in I\}$ is a Banach space. Let $C^{0}(I, I)$ denote the set of all continuous self-mappings on $I ; C^{0}(I, I)$ is a compact closed subset of $C^{0}(I)$.

The following lemma is useful in the proof of Theorem 3.2. 
Lemma 3.1 (see [12]). Let $i=1,2$, and suppose that $g_{i}$ is a self-homeomorphism of $I$ such that $\left|g_{i}(x)-g_{i}(y)\right| \leq N|x-y|$ for all $x, y \in I$, where $N>0$ is a constant. Then

(i) $\left\|g_{1}^{n}-g_{2}^{n}\right\| \leq\left(\sum_{i=0}^{n-1} N^{i}\right)\left\|g_{1}-g_{2}\right\|$ for all $n \in \mathbb{Z}$;

(ii) $\left\|g_{1}-g_{2}\right\| \leq N\left\|g_{1}^{-1}-g_{2}^{-1}\right\|$.

For $0 \leq l \leq L$, define

$$
\begin{aligned}
& \mathfrak{F}(I, l, L)=\left\{g \in C^{0}(I, I) \mid g(0)=0, g(1)=1, l(t-s) \leq g(t)-g(s) \leq L(t-s), \forall s \leq t \in I\right\}, \\
& \mathfrak{F}^{\prime}(I, l, L)=\left\{g \in C^{0}(I, I) \mid g(0)=1, g(1)=0, l(t-s) \leq g(s)-g(t) \leq L(t-s), \forall s \leq t \in I\right\} .
\end{aligned}
$$

Both $\mathfrak{F}(I, l, L)$ and $\mathfrak{F}^{\prime}(I, l, L)$ are compact convex subsets of $C^{0}(I)[1,13]$.

Theorem 3.2. Suppose (H1), (H2) hold and $F \in H_{1}^{0}\left(\mathbb{T}^{1},-1\right)$ with a Lipschitz constant $M>\alpha_{1}$. Equation (1.4) has a solution $f \in H_{1}^{0}\left(\mathbb{T}^{1},-1\right)$ with a Lipschitz constant $M / \alpha_{1}$.

Proof. Let $G$ and $\Psi$ be the induced maps of $F$ and $\Phi$ defined in Section 2. By Lemma 2.16 (1.4) is equivalent to the following equation:

$$
\Psi\left(g(t), g^{3}(t), \ldots, g^{2 n-1}(t)\right)=G(t), \quad t \in I
$$

By Lemma 2.3, the map $G \in C^{0}(I)$ is strictly decreasing $G(0)=1$, and $G(1)=0$. The map $G$ can be extended to a lift of $F$, and

$$
\left|G\left(t_{1}\right)-G\left(t_{2}\right)\right| \leq M\left|t_{1}-t_{2}\right|, \quad \forall t_{1}, t_{2} \in I
$$
operator

Let $M_{0}=\sum_{j=1}^{n} \beta_{j}\left(\alpha_{1}^{-1} M\right)^{2 j-2}$. Note that $\mathfrak{F}^{\prime}\left(I, 0, \alpha_{1}^{-1} M\right) \neq \phi$, since $M>\alpha_{1}$. Define an

$$
\mathfrak{L}: \mathfrak{F}^{\prime}\left(I, 0, \alpha_{1}^{-1} M\right) \longrightarrow C^{0}(I)
$$


by $g \mapsto \mathfrak{L}_{g}$, where

$$
\mathfrak{L}_{g}(t)=\Psi\left(t, g^{2}(t), \ldots, g^{2 n-2}(t)\right), \quad t \in I
$$

By (H2), we have for any $t \geq s \in I$,

$$
\begin{aligned}
\mathfrak{L}_{g}(t)-\mathfrak{L}_{g}(s) & =\Psi\left(t, g^{2}(t), \ldots, g^{2 n-2}(t)\right)-\Psi\left(s, g^{2}(s), \ldots, g^{2 n-2}(s)\right) \\
& \geq \alpha_{1}(t-s)+\sum_{j=2}^{n} \alpha_{j}\left(g^{2 j-2}(t)-g^{2 j-2}(s)\right) \\
& \geq \alpha_{1}(t-s), \\
\mathfrak{L}_{g}(t)-\mathfrak{L}_{g}(s) & =\Psi\left(t, g^{2}(t), \ldots, g^{2 n-2}(t)\right)-\Psi\left(s, g^{2}(s), \ldots, g^{2 n-2}(s)\right) \\
& \leq \beta_{1}(t-s)+\sum_{j=2}^{n} \beta_{j}\left(g^{2 j-2}(t)-g^{2 j-2}(s)\right) \\
& \leq M_{0}(t-s) .
\end{aligned}
$$

Hence, $\mathfrak{L}_{g}$ is increasing, $\mathfrak{L}_{g}(0)=0, \mathfrak{L}_{g}(1)=1$, and $\mathfrak{L}_{g} \in \mathfrak{F}\left(I, \alpha_{1}, M_{0}\right)$. Thus $\mathfrak{L}_{g}^{-1} \in \mathfrak{F}\left(I, M_{0}^{-1}\right.$, $\left.\alpha_{1}^{-1}\right)$.

Define $\mathfrak{T}: \mathfrak{F}^{\prime}\left(I, 0, \alpha_{1}^{-1} M\right) \rightarrow C^{0}(I)$ by

$$
\mathfrak{T}(g)=\mathfrak{L}_{g}^{-1} \circ G,
$$

where $\mathfrak{L}_{g}$ is defined in (3.5) and $G$ is the induced map of $F$. For convenience, denote $\mathfrak{T}(g)=$ $\mathfrak{T}_{g}$. Then, we have $\mathfrak{T}_{g}(0)=1, \mathfrak{T}_{g}(1)=0$. Since $\mathfrak{L}_{g}^{-1}$ is increasing, we have that for all $s, t \in I$ with $t \geq s$,

$$
\begin{aligned}
0 & \leq \mathfrak{T}_{g}(s)-\mathfrak{T}_{g}(t)=\mathfrak{L}_{g}^{-1} \circ G(s)-\mathfrak{L}_{g}^{-1} \circ G(t) \\
& \leq \alpha_{1}^{-1}(G(s)-G(t)) \leq \alpha_{1}^{-1} M(t-s) .
\end{aligned}
$$

Hence, $\mathfrak{T}(g) \in \mathfrak{F}^{\prime}\left(I, 0, \alpha_{1}^{-1} M\right)$. 
Furthermore, for any $g_{1}, g_{2} \in \mathfrak{F}^{\prime}\left(I, 0, \alpha_{1}^{-1} M\right)$, we have

$$
\begin{aligned}
\left\|\mathfrak{T}_{g_{1}}-\mathfrak{T}_{g_{2}}\right\| & =\left\|\mathfrak{L}_{g_{1}}^{-1} \circ G-\mathfrak{L}_{g_{2}}^{-1} \circ G\right\| \\
& =\left\|\mathfrak{L}_{g_{1}}^{-1}-\mathfrak{L}_{g_{2}}^{-1}\right\| \\
& \leq \alpha_{1}^{-1}\left\|\mathfrak{L}_{g_{1}}-\mathfrak{L}_{g_{2}}\right\|, \\
\left\|\mathfrak{L}_{g_{1}}-\mathfrak{L}_{g_{2}}\right\| & \leq \sup _{t \in I}\left|\Psi\left(t, g_{1}^{2}(t), \ldots, g_{1}^{2 n-2}(t)\right)-\Psi\left(t, g_{2}^{2}(t), \ldots, g_{2}^{2 n-2}(t)\right)\right| \\
& \leq \sum_{j=2}^{n} \beta_{j}\left\|g_{1}^{2 j-2}-g_{2}^{2 j-2}\right\| \\
& \leq \sum_{j=2}^{n} \beta_{j} \sum_{i=1}^{2 j-2}\left(\alpha_{1}^{-1} M\right)^{i-1}\left\|g_{1}-g_{2}\right\|,
\end{aligned}
$$

where Lemma 3.1 and (H2) are applied. Finally we get that

$$
\left\|\mathfrak{T}_{g_{1}}-\mathfrak{T}_{g_{2}}\right\| \leq \sum_{j=2}^{n} \beta_{j} \sum_{i=1}^{2 j-2} \alpha_{1}^{-i} M^{i-1}\left\|g_{1}-g_{2}\right\| .
$$

Hence $\mathfrak{T}$ maps $\mathfrak{F}^{\prime}\left(I, 0, \alpha_{1}^{-1} M\right)$ continuously into itself. By Schauder's fixed point theorem, $\mathfrak{T}$ has a fixed $g \in \mathfrak{F}^{\prime}\left(I, 0, \alpha_{1}^{-1} M\right)$. By Lemma 2.2, the map $g$ can be extended to a map $\tilde{g}$ such that $\tilde{g}(t+1)-\tilde{g}(t)=-1$, for all $t \in \mathbb{R}$, and there exists a unique $f \in C^{0}\left(\mathbb{T}^{1}, \mathbb{T}^{1}\right)$ such that $\tilde{g}$ is a lift of $f$. Note that for any $t, s \in \mathbb{R}$ with $t<s$, there is an integer $k$ and a nonnegative integer $m$ such that $t \in[k, k+1)$ and $s \in[k+m, k+m+1)$. Thus

$$
\begin{aligned}
|\tilde{g}(t)-\tilde{g}(s)| & \leq|\tilde{g}(t)-\tilde{g}(k+1)|+\sum_{j=1}^{m}|\tilde{g}(k+j)-\tilde{g}(k+j+1)|+|\tilde{g}(s)-\tilde{g}(k+m)| \\
& \leq|\tilde{g}(t-k)-\tilde{g}(1)|+(m-1)|\tilde{g}(0)-\tilde{g}(1)|+|\tilde{g}(s-k-m)-\tilde{g}(0)| \\
& \leq \frac{M}{\alpha_{1}}[1-(t-k)+m-1+s-k-m]=\frac{M}{\alpha_{1}}(s-t) .
\end{aligned}
$$

Obviously $f \in H_{1}^{0}\left(\mathbb{T}^{1},-1\right)$ and $f$ is a solution of (1.4) with a Lipschitz constant $M / \alpha_{1}$. 


\section{Uniqueness and Stability}

Let $F_{1}, F_{2} \in C^{0}\left(\mathbb{T}^{1}, \mathbb{T}^{1}\right)$ and $\widetilde{F}_{1}, \widetilde{F}_{2}$ be their lifts, respectively. By (iv) of Lemma 2.1, we can define

$$
\left\|F_{1}-F_{2}\right\|_{\mathbb{R}}:=\sup _{t \in \mathbb{R}}\left|\tilde{F}_{1}\left(t_{1}\right)-\tilde{F}_{2}\left(t_{2}\right)\right|
$$

Definition 4.1. For a given small constant $\epsilon>0$, one says that $F_{1}$ is $\epsilon-C^{0}$-close to $F_{2}$ if

$$
\left\|F_{1}-F_{2}\right\|_{\mathbb{R}}<\epsilon
$$

Now as in [8], we give the definition that (1.4) is stable.

Definition 4.2. Let $F_{1}, F_{2} \in C^{0}\left(\mathbb{T}^{1}, \mathbb{T}^{1}\right)$ and $f_{1}, f_{2}$ be corresponding solutions of (1.4) on $F_{1}, F_{2}$. One says that (1.4) is stable if for all $\varepsilon>0$ there is a $\delta>0$ such that if $F_{1}$ is $\delta-C^{0}$-close to $F_{2}$, then $f_{1}$ is $\varepsilon-C^{0}$ - close to $f_{2}$.

Theorem 4.3. Suppose that the conditions in Theorem 3.2 still hold, and

$$
r=\sum_{j=2}^{n} \beta_{j} \sum_{i=1}^{2 j-2} \alpha_{1}^{-i} M^{i-1}<1
$$

Then (1.4) has a unique solution $f \in H_{1}^{0}\left(\mathbb{T}^{1},-1\right)$ with a Lipschitz constant $M / \alpha_{1}$. Moreover, (1.4) is stable.

Proof. By Banach contraction theorem and (3.10), the solution $f$ is unique.

Suppose that $F_{1}, F_{2} \in H_{1}^{0}\left(\mathbb{T}^{1},-1\right)$ both satisfy the conditions in Theorem 3.2 and $f_{1}, f_{2}$ are the unique solutions of (1.4) corresponding to the given $F_{j}, j=1,2$, respectively. Assume that $\tilde{F}_{i}, \tilde{f}_{i}$ be the lifts of $F_{i}, f_{i}, i=1,2$, respectively. Let $G_{i}, g_{i}$ be the restrictions on $[0,1]$ of $\tilde{F}_{i}, \tilde{f}_{i}, i=1,2$. Let $\Psi$ be the induced map of $\Phi$ defined in (2.27). By the proof of Theorem 3.2, we have

$$
g_{i}=\mathfrak{L}_{g_{i}}^{-1} \circ G_{i}, \quad i=1,2
$$

where $\mathfrak{L}_{g_{i}}$ is defined as (3.5) and $\mathfrak{L}_{g_{i}}^{-1} \in \mathfrak{F}\left(I, M_{0}^{-1}, \alpha_{1}^{-1}\right), i=1$, 2. By (3.10), we have

$$
\begin{aligned}
\left\|g_{1}-g_{2}\right\| & =\left\|\mathfrak{L}_{g_{1}}^{-1} \circ G_{1}-\mathfrak{L}_{g_{2}}^{-1} \circ G_{2}\right\| \\
& \leq\left\|\mathfrak{L}_{g_{1}}^{-1} \circ G_{1}-\mathfrak{L}_{g_{1}}^{-1} \circ G_{2}\right\|+\left\|\mathfrak{L}_{g_{1}}^{-1} \circ G_{2}-\mathfrak{L}_{g_{2}}^{-1} \circ G_{2}\right\| \\
& \leq \alpha_{1}^{-1}\left\|G_{1}-G_{2}\right\|+r\left\|g_{1}-g_{2}\right\| .
\end{aligned}
$$

Thus

$$
\left\|g_{1}-g_{2}\right\| \leq\left[(1-r) \alpha_{1}\right]^{-1}\left\|G_{1}-G_{2}\right\|
$$


Discrete Dynamics in Nature and Society

For any $t \in \mathbb{R}$, there is an integer $k$ with $t \in[k, k+1)$ such that

$$
\left|\tilde{f}_{1}(t)-\tilde{f}_{2}(t)\right|=\left|\tilde{f}_{1}(t-k)+k-\tilde{f}_{2}(t-k)-k\right|=\left|g_{1}(t-k)-g_{2}(t-k)\right| .
$$

This means that

$$
\left\|\tilde{f}_{1}-\tilde{f}_{2}\right\|_{\mathbb{R}}=\left\|g_{1}-g_{2}\right\|
$$

By the same argument, we have

$$
\left\|\widetilde{F}_{1}-\widetilde{F}_{2}\right\|_{\mathbb{R}}=\left\|G_{1}-G_{2}\right\| .
$$

Thus

$$
\left\|\tilde{f}_{1}-\tilde{f}_{2}\right\|_{\mathbb{R}} \leq\left[(1-r) \alpha_{1}\right]^{-1}\left\|\tilde{F}_{1}-\tilde{F}_{2}\right\|_{\mathbb{R}}
$$

By the discussion above, we know that (1.4) is stable.

\section{Example}

Example 5.1. Consider the equation

$$
(f(z))^{359 / 360}\left(f^{3}(z)\right)^{(1 / 720 \pi i) \ln \left(f^{3}(z)\right)}=\exp \left(\frac{2 \pi i\left(e-z^{1 / 2 \pi i}\right)}{e-1}\right) .
$$

Obviously

$$
\begin{aligned}
& F(z)=\exp \left(\frac{2 \pi i\left(e-z^{1 / 2 \pi i}\right)}{e-1}\right) \\
& \Phi\left(z_{1}, z_{2}\right)=z_{1}^{359 / 360} z_{2}^{(1 / 720 \pi i) \ln \left(z_{2}\right)}
\end{aligned}
$$

The map $F$ has a lift $\tilde{F}$ and $\left.\widetilde{F}\right|_{[0,1]}=\left(e-e^{t}\right) /(e-1)$. Moreover, $F$ has a Lipschitz constant 2. The induced map of $\Phi$ is

$$
\begin{gathered}
\Psi\left(t_{1}, t_{2}\right)=\frac{359}{360} t_{1}+\frac{1}{360} t_{2}^{2}, \quad 0<t_{j}<1, j=1,2, \\
\Psi(0,0)=0, \quad \Psi(1,1)=1 .
\end{gathered}
$$

It is easy to verify that both (H1) and (H2) hold. The constants are $\alpha_{1}=\beta_{1}=359 / 360, \alpha_{2}=$ $0, \beta_{2}=1 / 180$. By Theorem 3.2, (5.1) has a solution $f \in H_{1}^{0}\left(\mathbb{T}^{1},-1\right)$ with a Lipschitz constant $720 / 359$. 
Note that $\alpha_{1}^{-1}=359 / 360<1$. We have

$$
r=\beta_{2} \sum_{i=1}^{2} \alpha_{1}^{-i} M^{i-1}<\frac{1}{180} \sum_{i=1}^{2} 2^{i-1}=\frac{1}{60} .
$$

Thus by Theorem 4.3, the solution is unique and the equation is stable.

\section{Acknowledgment}

The authors would like to thank the referees for their valuable comments and suggestions which led to truly significant improvement of the paper. This work is part of Project 11001064 supported by National Natural Science Foundation of China, Project HITC200706 and HITC200701 supported by Science Research Foundation in Harbin Institute of Technology.

\section{References}

[1] J. Zhang, L. Yang, and W. Zhang, "Some advances on functional equations," Advances in Mathematics, vol. 24, no. 5, pp. 385-405, 1995.

[2] K. Baron and W. Jarczyk, "Recent results on functional equations in a single variable, perspectives and open problems," Aequationes Mathematicae, vol. 61, no. 1-2, pp. 1-48, 2001.

[3] K. Ciepliński, "On the embeddability of a homeomorphism of the unit circle in disjoint iteration groups," Publicationes Mathematicae Debrecen, vol. 55, no. 3-4, pp. 363-383, 1999.

[4] W. Jarczyk, "Babbage equation on the circle," Publicationes Mathematicae Debrecen, vol. 63, no. 3, pp. 389-400, 2003.

[5] J. H. Mai, "Conditions for the existence of N-th iterative roots of self-homeomorphisms of the circle," Acta Mathematica Sinica, vol. 30, no. 2, pp. 280-283, 1987 (Chinese).

[6] P. Solarz, "On some iterative roots on the circle," Publicationes Mathematicae Debrecen, vol. 63, no. 4, pp. 677-692, 2003.

[7] M. C. Zdun, "On iterative roots of homeomorphisms of the circle," Bulletin of the Polish Academy of Sciences Mathematics, vol. 48, no. 2, pp. 203-213, 2000.

[8] M. C. Zdun and W. Zhang, "A general class of iterative equations on the unit circle," Czechoslovak Mathematical Journal, vol. 57, no. 3, pp. 809-829, 2007.

[9] M. Bajger, "On the structure of some flows on the unit circle," Aequationes Mathematicae, vol. 55, no. 1-2, pp. 106-121, 1998.

[10] L. S. Block and W. A. Coppel, Dynamics in One Dimension, vol. 1513 of Lecture Notes in Mathematics, Springer, Berlin, Germany, 1992.

[11] K. Ciepliński, "On properties of monotone mappings of the circle," Journal of Analysis and Applications, vol. 4, no. 3, pp. 169-178, 2006.

[12] W. N. Zhang, "Discussion on the differentiable solutions of the iterated equation $\sum_{i=1}^{n} \lambda_{i} F^{i}(x)=F(x)$," Nonlinear Analysis: Theory, Methods \& Applications, vol. 15, no. 4, pp. 387-398, 1990.

[13] W. Zhang, "Solutions of equivariance for a polynomial-like iterative equation," Proceedings of the Royal Society of Edinburgh A, vol. 130, no. 5, pp. 1153-1163, 2000. 


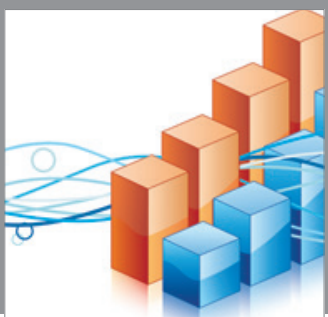

Advances in

Operations Research

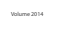

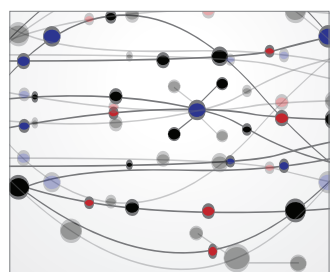

\section{The Scientific} World Journal
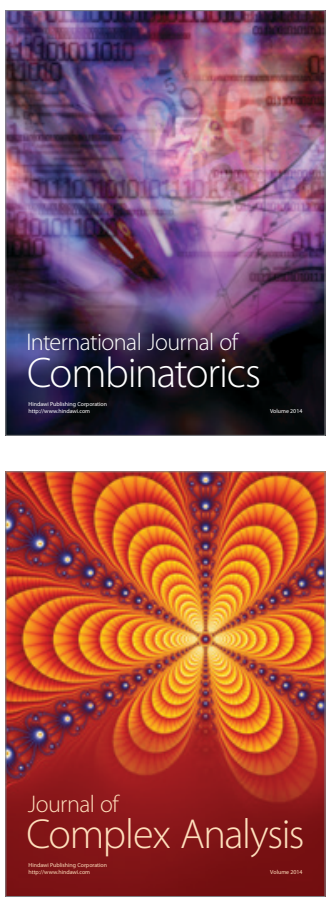

International Journal of

Mathematics and

Mathematical

Sciences
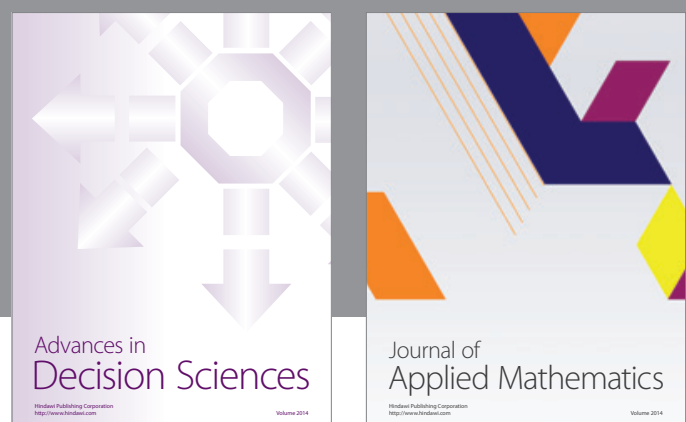

Journal of

Applied Mathematics
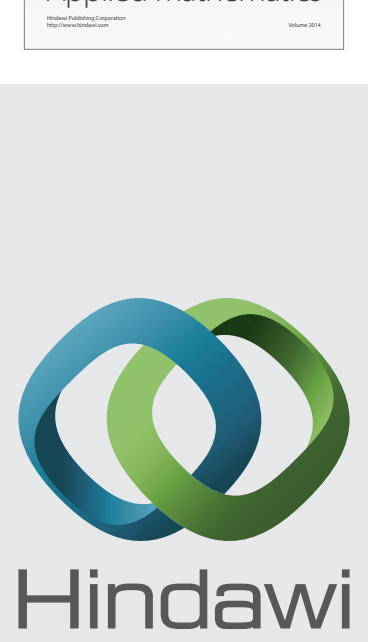

Submit your manuscripts at http://www.hindawi.com
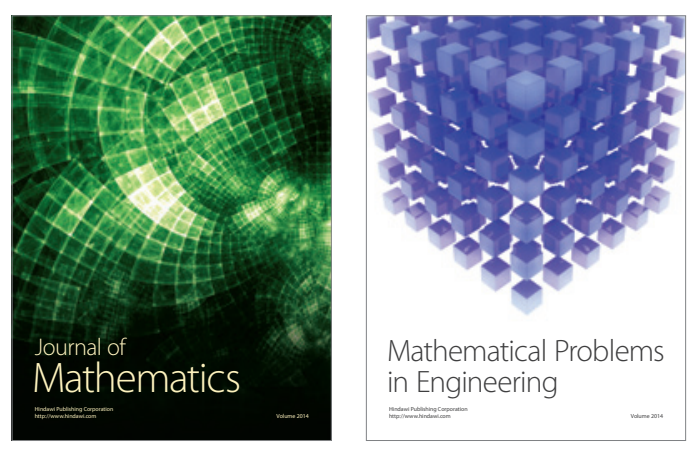

Mathematical Problems in Engineering
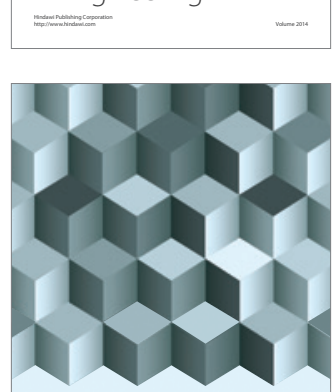

Journal of

Function Spaces
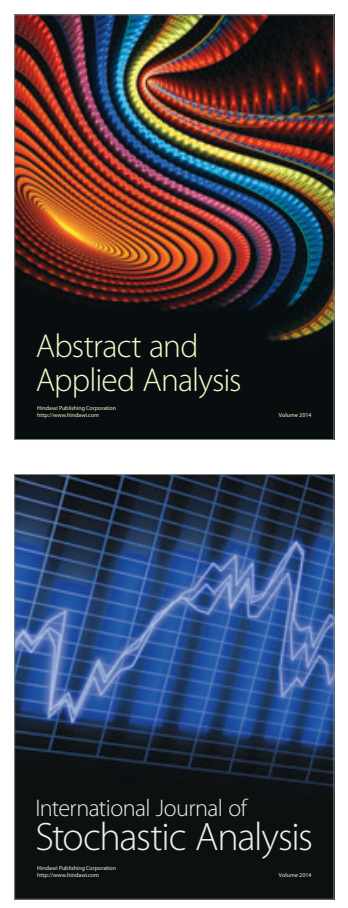

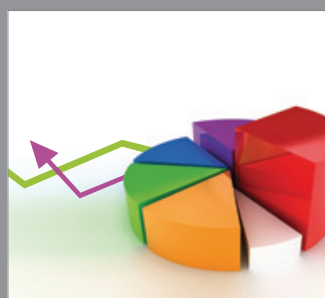

ournal of

Probability and Statistics

Promensencen
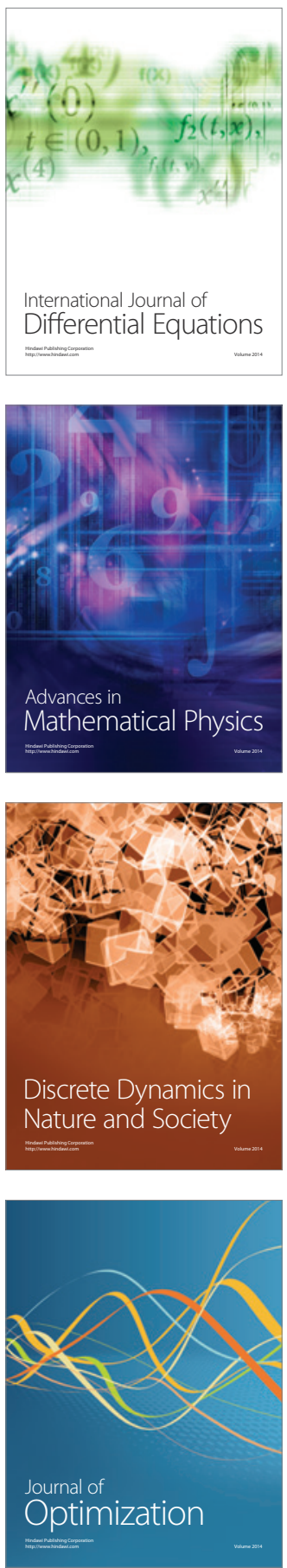